\title{
Role of water in sweet taste chemoreception*
}

\author{
Gordon G. Birch \\ School of Food Biosciences, The University of Reading, P.O. Box 226, \\ Whiteknights, Reading RG6 6AP, UK
}

\begin{abstract}
The mechanistic understanding of sweet taste chemoreception has been advanced by the microscopic and macroscopic studies of sweetener-water interactions. This approach has led to the concept of water mobility as a key to interpreting sweetness. The apparent specific volume of a solution is a determinant of its taste quality, as sweetness is known to be confined to the range $0.51-0.71 \mathrm{~cm}^{3} \mathrm{~g}^{-1}$. Thus, the "ideal" quality of the sugars is presumed to be due to their occupancy of the center of this range (i.e., $0.618 \mathrm{~cm}^{3} \mathrm{~g}^{-1}$ ). Most sweeteners elicit off-tastes and flavors and exhibit different apparent specific volumes. This leads to the conclusion that taste quality is broadly determined by the packing characteristics of sweet molecules among water molecules and the compactness of their hydration shells, expressed as their apparent specific isentropic compressibilities. The role of water can, therefore, be applied in modern attempts to optimize sweet taste quality, and different food salts can be explored as useful taste modifiers. Salts interact more strongly with water structure than do any other taste solutes, and it emerges that the ionic charge density is an important criterion. Such studies show how sweetener formulations are likely to improve within the next decade.
\end{abstract}

\section{INTRODUCTION}

Since the previous IUPAC conference on sweeteners, held in Jerusalem in 1996, studies of the role of water in sweet taste chemoreception have advanced prolifically and have included theoretical and practical approaches, microscopic and macroscopic effects, and hydrostatic and hydrodynamic mechanisms.

Because hydrogen bonding is important in the functioning of glucophores [1], as well as the structure of water [2], molecular dynamics simulations [3,4] have been used to compute the numbers of hydrogen bonds at specific sites of sugar rings in water, and the concept of "radial distribution function" has been used to calculate the likelihood of hydrogen-bond formation. Such theoretical approaches seem to lend some support to the experimental identification of AH,B sites in sugar molecules, but they must be interpreted with caution because they are all based on assumptions. For example, we need to be able to define when hydrogen-bond formation between sugar and water molecules actually begins [5].

Apparent specific volumes of tastants and their implications have been extensively explored during the past six years. However, these are only one aspect of hydration of sweetener molecules, and, more fundamentally, perturbation effects on water structure have been investigated at the University of Rheims. These have been based on spectroscopic measurements, such as Fourier transform infrared (FTIR) and laser-Raman spectroscopy, and they help to identify free -OH groups and changes in the amounts of "quasi-crystalline" or "amorphous" water species caused by the sweetener molecules.

\footnotetext{
*Pure Appl. Chem. Vol. 74, No. 7, 2002. A special topic issue on the science of sweeteners.
} 


\section{SPECTROSCOPIC STUDIES OF SWEETENERS IN WATER}

FTIR can be used to identify free -OH stretching signals, which can be used, in turn, to identify potential glucophores. This allows sweeteners (particularly those with sugar structures) to be compared. For example, FTIR shows big differences between sucrose and sucralose, and, indeed, sucrose shows more general similarities to fructose than to sucralose, which, ultimately, relates to taste quality characteristics.

Laser-Raman spectroscopy can directly examine effects on water structure, using a program to decompose the experimental Raman band. Small carbohydrates do not cause any noticeable change in water structure [6]. Only small changes are seen in $10 \% \mathrm{w} / \mathrm{v}$ solutions, which cause an overall structure-maker effect. Interestingly, sucralose Raman frequencies are shifted toward lower values, which is a sign of reinforced H-bonds in water. However, the integrated intensities of bands show a decrease of the quasi-crystalline water and an increase in the proportions of less-organized species of water structure (Table 1). Such observations lead Mathlouthi and Hutteau [6] to the conclusion that sweet taste intensity and enhancement are attributable to water mobility.

Table 1 Raman spectral bands of pure water and changes caused by sucralose [6].

\begin{tabular}{lccl}
\hline & $\begin{array}{c}\text { Component } \\
\text { wavelength band } \\
v\left(\mathrm{~cm}^{-1}\right)\end{array}$ & $\begin{array}{c}\text { Area } \\
\left(1_{\mathrm{e}}\right)\end{array}$ & \multicolumn{1}{c}{ Assignment } \\
{$[\%]$} & \\
\hline Water & 3239 & 64.1 & Quasi-crystalline phase \\
& 3414 & 20.8 & Solid-like amorphous phase \\
& 3537 & 13.5 & Liquid amorphous phase \\
Changes caused by & 3633 & 1.7 & Unassociated water molecules \\
sucralose in solution & -12 & -14 & Quasi-crystalline phase \\
& -6 & 13 & Solid-like amorphous phase \\
& -8 & -3 & Liquid amorphous phase \\
& -11 & 4 & Unassociated water molecules \\
\hline
\end{tabular}

\section{WATER MOBILITY AND DIFFERENT SWEETENERS}

Macroscopic parameters, such as intrinsic viscosity, [ $\eta]$, and surface tension are also good indicators of water mobility and have allowed Mathlouthi's group to examine many sweeteners in some detail. A low $[\eta]$ and a high Huggins' constant $\left(K^{\prime}\right)$ show high water mobility, and this might lead to enhancement of sweet taste. Likewise, high surface tension (e.g., measurable by a contact angle method) is observed for the salt-structured sweeteners (saccharin: Na; acesulphame: $\mathrm{K}$; and cyclamate: $\mathrm{Na}$ ), which indicates good compatibility with water structure, as with bulk sweeteners. Indeed, mixtures of bulk and salt-structured sweetness do frequently display good synergy [7,8] (Table 2). Among the simple sugars, D-fructose, with a cyclic methylene group, as a center of hydrophobicity, and a balancing center of high hydrophilicity, probably causes the greatest water mobility. It is, therefore, of interest to

Table 2 Physicochemical properties and synergy in binary sweetener mixtures [6].

\begin{tabular}{|c|c|c|c|}
\hline Sweetener mixture & $\begin{array}{c}\text { Synergy } \\
{[\%]}\end{array}$ & $\begin{array}{l}\text { Intrinsic viscosity, }[\eta] \\
\mathrm{cm}^{3} \mathrm{~g}^{-1}\end{array}$ & Huggins constant, $\mathrm{K}^{\prime}$ \\
\hline Sucrose - acesulphame K $(50: 50)^{\mathrm{a}}$ & 2 & 2.36 & 0.96 \\
\hline Sucrose - Na cyclamate $(50: 50)^{\mathrm{a}}$ & 11.8 & 2.28 & 1.23 \\
\hline Maltitol - acesulphame K $(50: 50)^{\mathrm{a}}$ & 18 & 2.38 & 1.16 \\
\hline Maltitol - Na cyclamate $(50: 50)^{\mathrm{a}}$ & 27.4 & 2.32 & 1.25 \\
\hline
\end{tabular}


identify the position of this hydrophilic center which, from field force calculations [9] and solution thermodynamics [10], appears to be the 3-4 $\alpha$-glycol group, i.e., the point furthest removed from the anomeric center. Fructose is the sweetest simple sugar on a weight basis.

Hydrophilicity, hydrophobicity, and water mobility all result from the particular structures of sweeteners and their interactions with water structure. The purity of sweet taste displayed by the sugars presumably originates in these interactions, as well as the unique hydrophilicity of the sugar structure. Sugars all possess an anomeric center or a masked (substituted) anomeric center, and it is noteworthy that this center is not directly involved in initiating the sweetness response.

\section{APPARENT SPECIFIC VOLUMES AND EFFECTS OF THE ANOMERIC CENTER}

Apparent specific volumes (ASVs) are hydrostatic packing volumes of molecules that have been previously reported to be crucially important in taste chemistry [10,11]. In the field of sweeteners, they are measured in water, at concentrations relating to actual tasting, by precision densitometry, and they have been shown to be broad determinants of taste quality [10]. Fundamentally, apparent specific volumes are the resultants of displacement and electrostrictive effects, and they correlate very well with theoretical measurements [12]. The fact that apparent specific volumes do not alter much when changes of configuration occur at the anomeric center $[10,13]$ underlines the finding that the anomeric center of sugars is not a hydrophilic site [9] and does not, therefore, interact directly with the sweet receptor. When monosaccharides are hydrogenated (loss of anomeric center), however, their ASVs increase by about $10 \%$, indicating lower packing efficiency among water molecules [14]. This seems to refute the idea that the anomeric center is hydrophobic. However, the increase in apparent volume upon hydrogenation is actually due to loss of cyclic structure because inositol (no anomeric center) has a lower apparent specific volume than the hexoses [10]. Moreover, Lopez-Chavez and Birch [14] have neatly shown that the apparent specific volumes of hydrogenated disaccharides are actually lower than their parent sugars, and they are sweeter (Fig. 1).

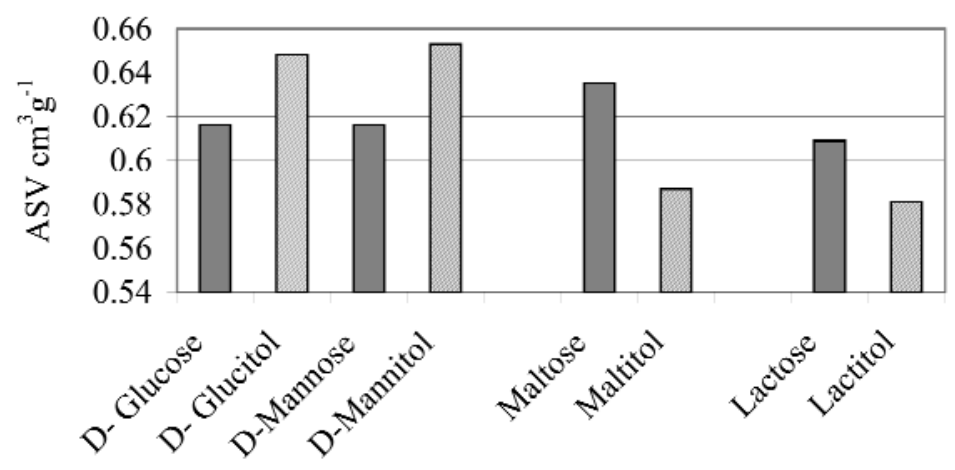

Fig. 1 Effects of hydrogenation on ASVs of sugars.

Since 1996, many ASV measurements of tastants have been made [15,16], and the idea of ASV as a broad determinant of taste quality has been confirmed (Fig. 2). ASV is one of several solution thermodynamic parameters that have been used to elucidate the mechanisms of human chemical senses [17] and drug efficiency [18]. 


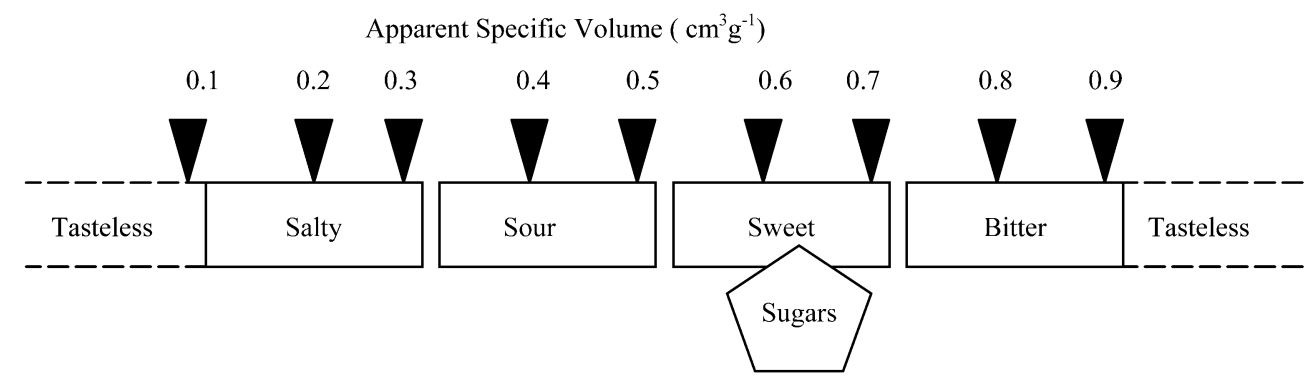

Fig. 2 The human "taste taste" spectrum by ASV [10].

\section{APPARENT SPECIFIC VOLUME AND GLUCOPHORES}

The ASV hypothesis augments the Shallenberger and Acree [1] AH,B glucophore hypothesis. In other words, ASV only determines taste quality for molecules with an appropriate sapophore. This explains certain anomalies, such as why certain molecules with many potential glucophores (e.g., the gluconates) do not taste sweet. The gluconate anion has an ASV of $0.58 \mathrm{~cm}^{3} \mathrm{~g}$, which places it in the sweet range. However, the cation (e.g., $\mathrm{Na}^{+}$) that makes a negative contribution to ASV, brings the value down. The ASV of sodium gluconate is, therefore, $0.49 \mathrm{~cm}^{3} \mathrm{~g}^{-1}$, which is in the acid range. However, the molecule has no free protons and is therefore tasteless, which makes it useful as a taste modifier (see below).

\section{COMPRESSIBILITY AND CHANGING TASTE QUALITY}

As a consequence of the packing of the solute molecule among water molecules, the solute becomes hydrated, and the ensuing electrostrictive forces determine the compaction of the hydration layer. This is reflected in the apparent specific isentropic compressibility $(\mathrm{K} 2 \mathrm{~s})$, a parameter that is determinable from density-sound measurements [19]. K2s often shows the same trends among tastants, as does ASV, since a molecule that packs well among water molecules is usually well hydrated. However, the parameters describe different roles of the solute in water, and these may turn out to be important in taste perception. For example, most cations contribute negatively to ASV, whereas most anions contribute positively. On the other hand, both anions and cations usually have negative effects on K2s. These distinctions provide guidelines for modern attempts to improve sweet taste quality.

The concentration of a solute in water may, of itself, affect taste quality by altering apparent specific volume. This is because apparent specific volume is not constant, but generally increases with increasing concentration. However, even when concentration of the solute remains constant, autohydrolysis may alter taste quality over time. The best-known example of this [16] is D-glucono 1,5-lactone, which changes from sweet to sour within $30 \mathrm{~min}$ of dissolution. During this time, the corresponding solution chemistry changes are accurately determinable, and measurements of apparent specific isentropic compressibility, ASV, and specific rotation provide parallel objective recordings of the taste changes.

\section{ROLE OF WATER IN SWEET TASTE MODULATION}

The role of water in sweet taste modulation [20] can be harnessed to advantage by food and pharmaceutical chemists in order to overcome the many negative attributes of the small number of sweeteners permitted for food use. These negative attributes include persistence, bitterness, and "off-flavors" and can often be characterized by known solution effects. On the basis that taste quality is broadly determined by ASV, the ASV of total solute can be altered by adventitious solutes, which interact strongly with water structure and alter taste perception accordingly. Solution measurements already indicate 
salts as the best agents for this, but the difficulty in this type of taste modulation lies in selecting an effective salt that does not in itself contribute any taste or flavor. One such ideal candidate is sodium gluconate, a permitted food ingredient with a bland taste that can be usefully employed in dental preparations (e.g., mouthwashes) to decrease bitter effects. Salts, such as sodium gluconate, act by depressing ASV of total solute, thus minimizing the possibility of bitterness. The gluconate anion (like most anions) makes a positive contribution to ASV. It is, therefore, advantageous to choose a strongly positively charged cation (e.g., $\mathrm{Mg}^{2+}$ ) to derive the greatest possible negative effect on ASV. However, the cationic charge is not the determining factor. Rather, it is the charge density, which in turn depends on the ionic radius [21]. Aroulmoji et al. [22] have recently described the marked advantage of magnesium gluconate in modifying the solution behavior of sucrose and have used low-resolution pulse NMR measurements to explain the motional properties of water in sugar-gluconate solutions.

\section{SYNERGY}

Sweetness synergy is a controversial subject [23], and reported examples are clearly dependent on the method by which the synergy is evaluated [24,25]. Reported magnitudes of synergy never seem to reach $100 \%$, and there is no accepted mechanistic explanation of the phenomenon. One possibility is that, in a binary sweetener mixture, one solute alters water structure, which then affects the solution behavior of the other solute, thus improving access to and/or action on receptors. Some of the best-studied (and most positive) examples of synergy are with mixtures of intense and bulk sweeteners, and maltitol with sodium cyclamate provides a clear illustration of this (27.4\% synergy, Table 2). Table 3 illustrates the massive change in apparent specific isentropic compressibility caused by the presence of sodium cyclamate in maltitol solution, which may ultimately be responsible for the synergistic effect.

Table 3 Apparent isentropic compressibility $\left[\mathrm{K}_{2(\mathrm{~s})}\right]$ of maltitol $(7.12 \% \mathrm{w} / \mathrm{v})$ + Na cyclamate (variable).

\begin{tabular}{cc}
\hline $\begin{array}{c}\text { Concentration of } \\
\text { Na cyclamate }(\% \mathrm{w} / \mathrm{v})\end{array}$ & $\begin{array}{c}\mathrm{K}_{2(\mathrm{~s})} \mathrm{cm}^{3} \mathrm{~g}^{-1} \mathrm{bar}^{-1} \\
\left(\times 10^{-8}\right)\end{array}$ \\
\hline 0.44 & -7.48 \\
0.3 & -6.97 \\
0.2 & -6.51 \\
0.1 & -6.27 \\
0.0 & -5.57 \\
\hline
\end{tabular}

\section{CONCLUSION}

Many aspects of the taste quality and effectiveness of sweet molecules are now interpretable by solution behavior, and it seems that the role of water in mediating such effects is indisputable. Water stabilizes, solvates, and transports tastant molecules to receptors, and solution measurements clearly illustrate differences between molecules in relation to their tastes. The explanation of taste behavior by molecular hydration mechanisms is applicable to many of the problems in the modern food and pharmaceutical industries.

\section{ACKNOWLEDGMENT}

This study has been carried out with financial support from the Commission of the European Communities, Agriculture, and Fisheries (FAIR) specific concerted action program CT98-4040 "The 
Optimisation of Sweet Taste Quality". It does not reflect its views and in no way anticipates the Commission's future policy in this area.

\section{REFERENCES}

1. R. S. Shallenberger and T. E. Acree. Nature 216, 480-482 (1967).

2. F. Franks. Pure Appl. Chem. 59, 1189-1202 (1987).

3. T. Astley, G. G. Birch, M. G. B. Drew, P. M. Rodger, G. R. H. Wilden. Food Chem. 56, 231-240 (1996).

4. T. Astley, G. G. Birch, M. G. B. Drew, P. M. Rodger, G. R. H. Wilden. J. Comput. Chem. 19, 363-367 (1998).

5. T. Astley, G. G. Birch, M. G. B Drew, P. M. Rodger. J. Phys. Chem. 103, 5080-5090 (1999).

6. M. Mathlouthi and F. Hutteau. Food Chem. 64, 77-82 (1999).

7. F. Hutteau, M. Mathlouthi, M. O. Portmann, D. Kilcast. Food Chem. 63, 9-16 (1998).

8. S. A. Parke, G. G. Birch, M. O. Portmann, D. Kilcast. Food Chem. 67, 247-260 (1999).

9. F. W. Lichtenthaler and S. Immel. Sweet-taste Chemoreception, M. Mathlouthi, G. G. Birch, J. A. Kanters (Eds.), pp. 21-54, Elsevier Applied Science, Oxford (1993).

10. S. Shamil, G. G. Birch, M. Mathlouthi, M. N. Clifford. Chem. Senses 12, 397-409 (1987).

11. G. G. Birch. Trends Food Sci. Tech. 7, $403-407$ (1996).

12. W. J. Spillane, G. G. Birch, M. G. B. Drew, I. Bartolo. J. Chem. Soc. Perkin Trans. 2 4, 497-503 (1992).

13. G. G. Birch, S. Shamil, Z. Shepherd. Experientia 42, 1232-1234 (1986); A. Lopez-Chavez and G. G. Birch. Chem. Senses 22 149-161 (1997).

14. S. A. Parke and G. G. Birch. J. Agric. Food Chem. 47, 1378-1384 (1999).

15. S. A. Parke, G. G. Birch, D. B. MacDougall, D. A. Stevens. Chem. Senses 22, 53-65 (1997).

16. Y. Yamanaka. Chem. Senses 21 (4), 405-409 (1996).

17. J. C. McGowan and A. Mellors. Molecular Volumes in Chemistry and Biology: Applications Including Partitioning and Toxicity, E. Horwood, Halsted Press, New York, Chichester (1986).

18. S. A. Galema and H. Hoiland. J. Phys. Chem. 95, 5321-5326 (1991).

19. G. G. Birch. Biofactors 9, 73-8 (1999).

20. Y. Markus. Ion Properties, p. 259, Marcel Dekker, New York (1997).

21. V. Aroulmoji, M. Mathlouthi, G. G. Birch. Food Chem. 70, 471-482 (2000).

22. G. G Birch, S. A. Parke, R. Siertsema, J. M. Westwell. Pure Appl. Chem. 69, 685-692 (1997).

23. S. S. Schiffman, B. J. Booth, B. T. Carr, M. L. Losee, E. A. Sattely-Miller, B. G. Graham. Brain Res. Bull. 38, 105-120 (1995).

24. S. S. Schiffman, E. A. Sattely-Miller, B. G. Graham, B. J. Booth, K. Giles. Chem. Senses 25, $131-140$ (2000). 